\title{
Using osmotic stress to stabilize mannitol production in Synechocystis sp. PCC6803
}

Wenyang Wu' ${ }^{1}$, Wei Du ${ }^{1}$, Ruth Perez Gallego ${ }^{2,3}$, Klaas J. Hellingwerf ${ }^{1,2}$, Aniek D. van der Woude ${ }^{2 \dagger}$ and Filipe Branco dos Santos ${ }^{1 *+}$

\begin{abstract}
Background: Mannitol is a C(6) polyol that is used in the food and medical sector as a sweetener and antioxidant, respectively. The sustainable production of mannitol, especially via the direct conversion of $\mathrm{CO}_{2}$ by photosynthetic cyanobacteria, has become increasingly appealing. However, previous work aiming to achieve mannitol production in the marine Synechococcus sp. PCC7002 via heterologous expression of mannitol-1-phosphate-5-dehydrogenase $(m t / D)$ and mannitol-1-phosphatase ( $m 1 p$, in short: a'mannitol cassette'), proved to be genetically unstable. In this study, we aim to overcome this genetic instability by conceiving a strategy to stabilize mannitol production using Synechocystis sp. PCC6803 as a model cyanobacterium.

Results: Here, we explore the stabilizing effect that mannitol production may have on cells faced with osmotic stress, in the freshwater cyanobacterium Synechocystis sp. PCC6803. We first validated that mannitol can function as a compatible solute in Synechocystis sp. PCC6803, and in derivative strains in which the ability to produce one or both of the native compatible solutes was impaired. Wild-type Synechocystis, complemented with a mannitol cassette, indeed showed increased salt tolerance, which was even more evident in Synechocystis strains in which the ability to synthesize the endogenous compatible solutes was impaired. Next we tested the genetic stability of all these strains with respect to their mannitol productivity, with and without salt stress, during prolonged turbidostat cultivations. The obtained results show that mannitol production under salt stress conditions in the Synechocystis strain that cannot synthesize its endogenous compatible solutes is remarkably stable, while the control strain completely loses this ability in only 6 days. DNA sequencing results of the control groups that lost the ability to synthesize mannitol revealed that multiple types of mutation occurred in the $m t / D$ gene that can explain the disruption of mannitol production.

Conclusions: Mannitol production in freshwater Synechocsytis sp. PCC6803 confers it with increased salt tolerance. Under this strategy, genetically instability which was the major challenge for mannitol production in cyanobacteria is tackled. This paper marks the first report of utilization of the response to salt stress as a factor that can increase the stability of mannitol production in a cyanobacterial cell factory.
\end{abstract}

Keywords: (D-)Mannitol, Synechocystis sp. PCC6803, Compatible solutes, Production stability, Salt stress

*Correspondence: f.brancodossantos@uva.nl

${ }^{\dagger}$ Aniek D. van der Woude and Filipe Branco dos Santos contributed equally to this work

${ }^{1}$ Molecular Microbial Physiology Group, Swammerdam Institute for Life Sciences, Faculty of Science, University of Amsterdam, Science Park 904, 1098 XH Amsterdam, The Netherlands

Full list of author information is available at the end of the article

\section{Background}

Mannitol is a six-carbon sugar alcohol with multiple biological applications, such as a sweetener and an antioxidant. Therefore, mannitol has been widely applied in the food-, pharmaceutical-, and chemical industry, and it is of high commercial value [1]. Recently, cyanobacteria have gained much attention to be developed as photosynthetic cell factories to convert $\mathrm{CO}_{2}$ directly into biochemical 
compounds of interest. Enabled by genetic engineering, the production of many different compounds [2-8] (e.g., ethanol, lactate, terpenes to name only a few) has already been achieved in different cyanobacterial species. Given the commercial value of mannitol and our current need for green alternatives, sustainable production of this sweetener by cyanobacteria is therefore becoming increasingly attractive.

The first report of mannitol production directly from $\mathrm{CO}_{2}$ in the marine cyanobacterium Synechococcus.sp PCC7002 (hereafter, Synechococcus) appeared in 2014 [9]. This production was achieved via heterologous expression of the genes encoding mannitol-1-phosphate5-dehydrogenase $(m t l D)$ and mannitol-1-phosphatase ( $m 1 p)$ either as individual proteins [9] or as a fused protein [10], to convert (part of) the endogenous metabolite fructose-6-phosphate into mannitol. This two-step conversion from fructose-6-phosphate to mannitol is in principle superior to the single-step conversion of fructose to mannitol via mannitol dehydrogenase $(m d h)$. This is due to the fact that in cyanobacteria, fructose-6-phosphate is more abundant comparing to fructose, since fructose6-phosphate is one of the main metabolites in the Pentose Phosphate Pathway that carries a high metabolic flux under photoautotrophic conditions. Hence, more mannitol would be expected to be produced via this two-step conversion, though the accumulation of the intermediate mannitol-1-phosphate might be harmful for the cells resulting in genetic instability problems [11]. In the study of Jacobsen and Frigaard, a concentration of mannitol of $1.1 \mathrm{~g} \mathrm{~L}^{-1}$ was reached after 12 days, with an average productivity of $0.15 \mathrm{~g} \mathrm{~L}^{-1} \mathrm{day}^{-1}$ [9]. However, this production system turned out to be genetically unstable, possibly because the heterologous mannitol production pathway directly competes for metabolic intermediates with biomass formation [12], which imposes a fitness burden on the mannitol producing cells. These strains thus become susceptible to suppressor mutations, such as insertions or deletions, that would lower/remove this fitness burden. In the study of Jacobsen and Frigaard [9], the genetically engineered Synechococcus strains suffered from both incomplete genome segregation and from suppressor mutations occurring in the $m t l D$ locus, which clearly indicates the genetic instability of mannitol production in those strains.

By aligning product formation to biomass synthesis, growth-coupled production promises to become a useful strategy to stabilize production from $\mathrm{CO}_{2}$ in cyanobacteria [13]. To implement this strategy, product formation needs to be either beneficial to the cells, or become a mandatory process for biomass synthesis. Under such conditions, Darwinian selection will ensure that the producing cells will not be outcompeted by non-producing mutant cells, i.e., the production would be stabilized. Before such rationale can be applied to mannitol production in cyanobacteria, we first want to understand the function that mannitol plays inside the cells that are able to produce this compound. According to literature, mannitol can serve as a compatible solute in selected bacteria, presumably because it will protect cells under high-salt- and/or oxidative stress conditions [14]. Since cyanobacteria natively produce various compatible solutes (e.g., trehalose, glycine betaine, sucrose, glucosyl-glycerol, etc.) to accommodate the consequences of salt stress $[15,16]$, we want to first experimentally validate whether mannitol can be used to replace the cyanobacterial native compatible solutes under salt stress conditions. If so, mannitol production would be expected to be beneficial for cyanobacterial cells that lack the endogenous compatible solutes in their resistance to salt stress. A mannitol production system would hence be expected to be stable in such cyanobacterial mutants under salt stress conditions.

To implement this idea, the model freshwater cyanobacterium Synechocystis sp. PCC6803 (hereafter, Synechocystis) was chosen over Synechococcus for a few reasons. In addition to being extensively genetically engineered for the production of a variety of biofuels and chemicals [4], Synechocystis is the only cyanobacterium in which the molecular mechanism of salt stress has been studied in detail [17]. The genes and biosynthetic pathways of its native compatible solutes (i.e., sucrose and glycosyl-glycerol) under salt stress have been clarified [18], which facilitates their targeted deletion and provides a straightforward approach to introduce a 'mannitol cassette' ( $m t l D$ and $m 1 p)$. Furthermore, the freshwater Synechocystis has a much higher sensitivity to salt stress than the marine Synechococcus $[19,20]$. During high salt acclimation, one would therefore expect more mannitol to be produced in Synechocystis than in Synechococcus, provided salt tolerance increases with cellular mannitol production. Given these reasons, we have selected Synechocystis as the microbial host to be genetically engineered for mannitol production.

In this study, we achieved mannitol production in Synechocystis via heterologous gene expression of a cassette composed of the codon-optimized mannitol-1-phosphate-5-dehydrogenase ( $m t D)$ from E. coli and mannitol-1-phosphatase $(m 1 p)$ from Eimeria tenella. We have further shown that mannitol can indeed function as a compatible solute, to benefit cell growth especially for the Synechocystis mutant strains that have lost the ability to synthesize their endogenous compatible solute(s). Significantly, by adding salt to the growth media, this strategy has been proven capable of stabilizing mannitol 
production during prolonged cultivations in these latter Synechocystis strains.

\section{Results and discussion}

\section{Engineering mannitol-producing Synechocystis strains}

It has been reported that mannitol can be synthesized from fructose-6 phosphate via the sequential enzymatic reactions catalyzed by mannitol-1-phosphatedehydrogenase $(m t l D)$ and mannitol-1-phosphatase ( $m 1 p)$ (Fig. 1). All our efforts to construct plasmids containing a functional mannitol cassette under control of the strong constitutive Ptrc1 promoter (a hybrid between the trp and lac UV5 promoters) have failed in E. coli, presumably due to the toxicity of certain sugar phosphates [11]. For instance, E. coli mutants that lose the function of fructose-1-phosphate dehydrogenase,

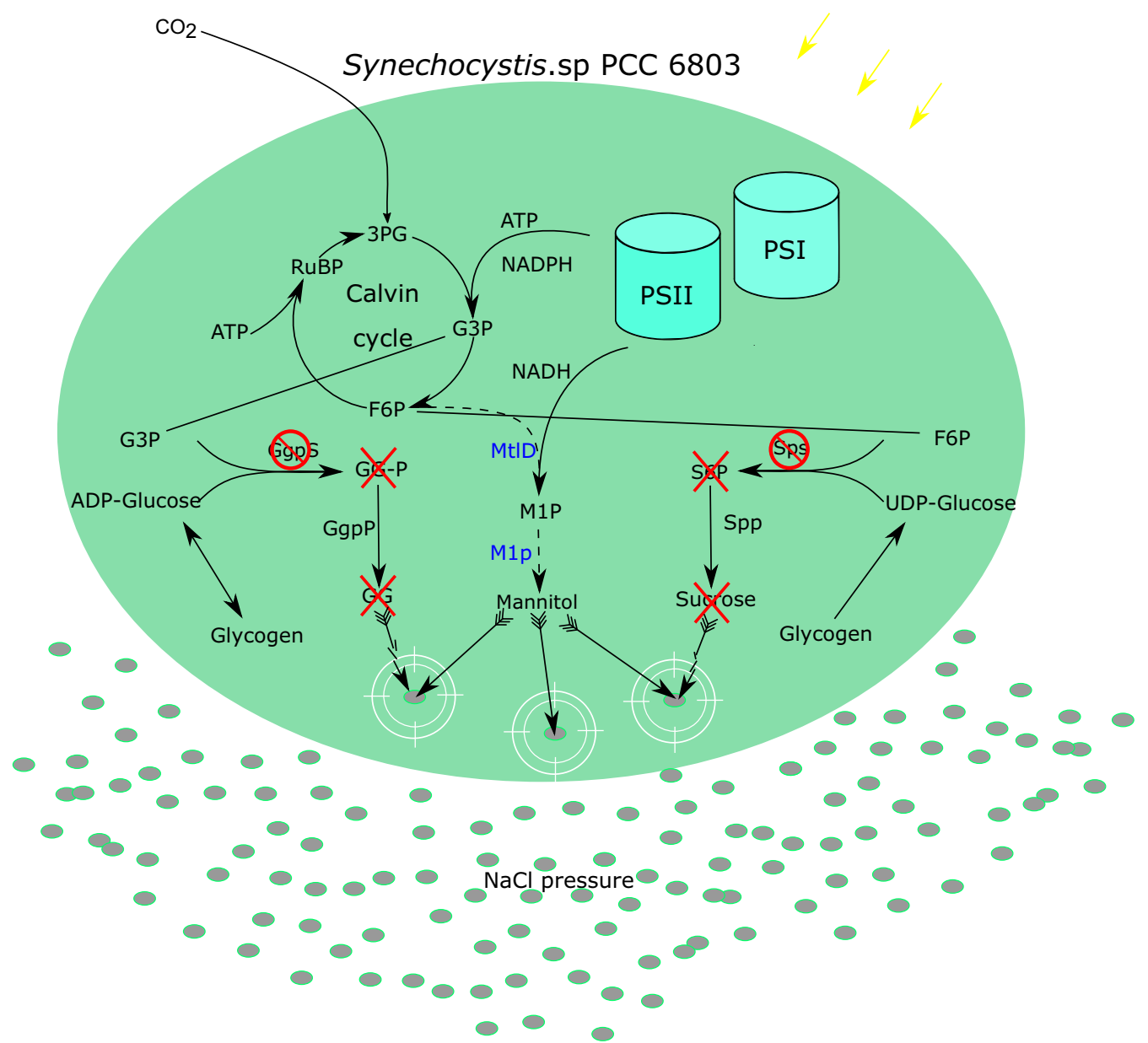

Fig. 1 Overview of the engineered biosynthetic pathway to mannitol in the cyanobacterium Synechocystis sp. PCC6803. Solid arrows without wings represent native steps in cell; dashed arrows represent introduced pathway of mannitol synthesis; engineered enzymes are shown in blue; red circles with diagonal red line indicate deleted proteins; red cross-lines indicate metabolites that cannot be synthesized. White double circles represent protection against osmotic pressure by compatible solutes or mannitol in the cells. Grey ovals represent salt stress. 3PG, 3-phosphoglycerate; F6P, fructose-6-phosphate; G3P, glyceraldehyde-3-phosphate; M1P, mannitol-1-phosphate; M1p, Mannitol-1-phosphatase (M1Pase; encoded by m1p from E.tenella); MtID, Mannitol-1-phosphate dehydrogenase (M1PDH; encoded by mtID from E. coli); GgpS,

Glucosyl-glycerol phosphate synthase; GG-P, glucosyl-glycerol-phosphate; GgpP, Glucosylglycerol phosphate phosphatase; GG, glucosyl-glycerol; Sps, Sucrose phosphate synthase; S6P, sucrose-6-phosphate; Spp, Sucrose phosphate phosphatase; RuBP, ribulose-1,5-bisphosphate 
via mutagenesis, have impaired cell growth due to the intracellular accumulation of fructose-1-phosphate [21]. A similar phenomenon was also observed in Salmonella typhimurium, when mannitol was added to the growth medium, cells that lost the function of mannitol dehydrogenase could not grow because of the intracellular accumulation of mannitol-1-phosphate [22]. Hence, instead of using an intact plasmid, a fused and linear DNA fragment, consisting of the homologous regions of slr0168 (a non-essential hypothetical protein), the mannitol production cassette and a kanamycin resistance cassette, was constructed to be integrated via natural transformation at the neutral site slr0168 of the Synechocystis genome [23]. After several attempts, we obtained a few positive colonies, though a methionine was always stubbornly missing at position 332 of $m t l D$ (even though not present in the linear DNA fragment used for the transformation). Nonetheless, since even with this mutated $m t l D$, mannitol production could still be observed, we decided to continue with this construct for the subsequent experiments.
This mannitol cassette was expressed in the wild type (WT), $\Delta g g p S$ ( $\triangle \mathrm{GGPS}$ ) and $\Delta g g p S \Delta s p s(\Delta \mathrm{CS})$ Synechocystis backgrounds, resulting in the strains: WT_M, $\triangle$ GGPS_M and $\triangle \mathrm{CS} \_\mathrm{M}$, respectively (Fig. 2a, b and Additional file 1: Table S1). Compared with a previous study in Synechococcus [9], this mutated mannitol cassette seems much easier to fully segregate, which occurs for the slr0168 neutral site of Synechocystis in only 5 days under $50 \mu \mathrm{g} \mathrm{mL}{ }^{-1}$ kanamycin. This could be influenced by the lower expression and/or activity level of MtlD due to the mutation on position 332. Next, we monitored the growth of each mutant in regular BG-11 medium. Growth of the strains with a functional mannitol cassette appeared clearly impaired (Fig. 3a). This is due to the burden of mannitol production, for instance because part of the carbon fixed in photosynthesis is not available for biomass formation. Strikingly, impairing the ability to synthesize glycosyl-glycerol, or both sucrose and glycosyl-glycerol, did not lead to any improvement in mannitol production; it even had a negative impact relative to the Synechocystis WT background (Fig. 3b and Additional file 1: Table S1).

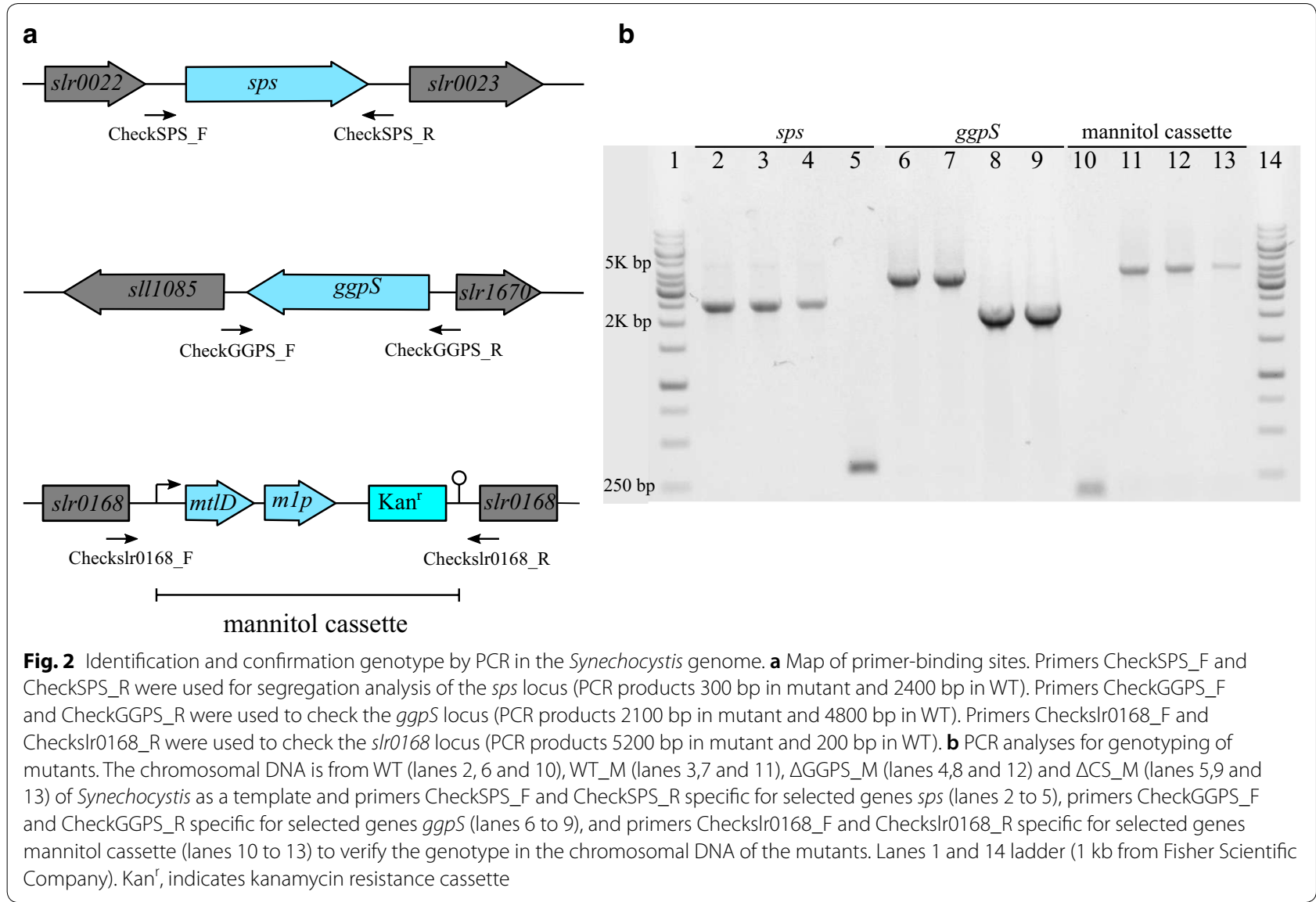


a

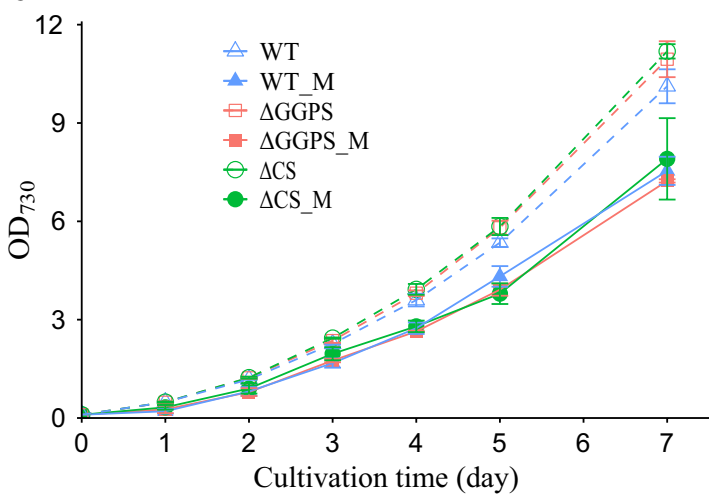

b

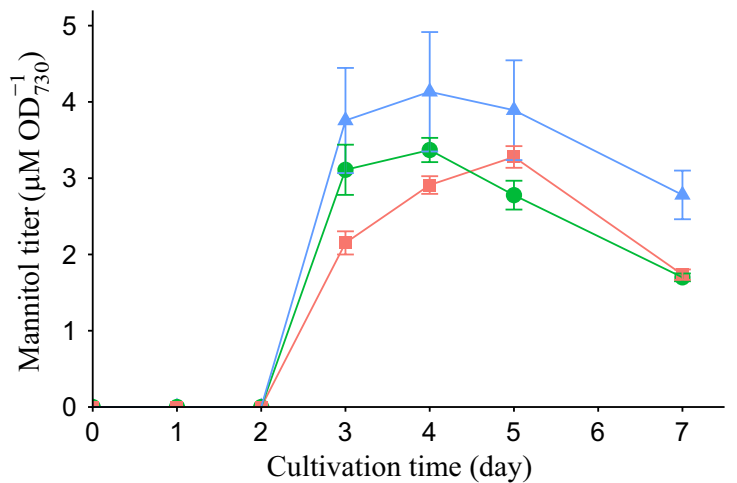

Fig. 3 Growth curve and mannitol production of Synechocystis mutants in normal BG-11 medium. a Growth curve in cultures of WT, WT_M, $\triangle C S$, $\triangle C S \_M, \triangle G G P S$ and $\triangle$ GGPS_M b Mannitol accumulation in three different mannitol-producing strains WT_M, $\triangle C S \_M$ and $\triangle$ GGPS_M. Symbols: solid triangles, WT_M; solid squares, $\triangle G G P S \_M$; solid circles, $\triangle C S \_M$; open triangles, WT; open squares, $\triangle G G P S$; open circles, $\triangle C S$. Values represent the average of at least three biological replicates (error bars represent standard deviation). In a, the broken lines connect the data points of the strains lacking the mannitol cassette

\section{Mannitol production confers cells with higher salt tolerance}

To test whether mannitol can functionally replace the native compatible solutes of Synechocystis to resist highsalt stress, the salt sensitivity of the WT, $\triangle$ GGPS and $\Delta \mathrm{CS}$ mutants, in the absence and presence of the mannitol cassette, was assayed under a wide range of salt stress conditions via a spot assay. Linear gradient $\mathrm{NaCl}$ plates were prepared with a salt gradient from 0 to $1 \mathrm{M}$ for the WT and $\triangle$ GGPS strains, and from 0 to $0.5 \mathrm{M}$ for the $\Delta \mathrm{CS}$ strain. The two salt gradients were used because the $\Delta C S$ strain is much more sensitive to increasing salt concentrations than the other two strains. This is to such an extent that the salt tolerance conferred by mannitol production can in fact only be clearly noticed for the $\Delta C S$ strain when the concentrations are between 0 and $0.5 \mathrm{M}$. Next, exponentially growing cultures of all strains were diluted to a concentration of 12,500 cells $\mu \mathrm{L}^{-1}$ and $5 \mu \mathrm{L}$ of each strain was spotted multiple times across the salt gradient on the BG-11 plate. The two different salt gradient plates were incubated at $30{ }^{\circ} \mathrm{C}$ under a constant moderate light intensity of $\sim 50 \mu \mathrm{mol}$ photons $\mathrm{m}^{-2} \mathrm{~s}^{-1}$. After about 1 week, the spots became green and the results were then analyzed.

All the strains carrying a mannitol cassette displayed increased salt tolerance (Fig. 4). In the Synechocystis WT background, sucrose and glycosyl-glycerol are the two main endogenous compatible solutes that are used by the cells to cope with high salt stress. It is quite interesting to see that even with the native compatible solutes present in the cell, mannitol production still confers cells even higher salt tolerance in the WT background (see top panel of Fig. 4). When the biosynthetic capacity for

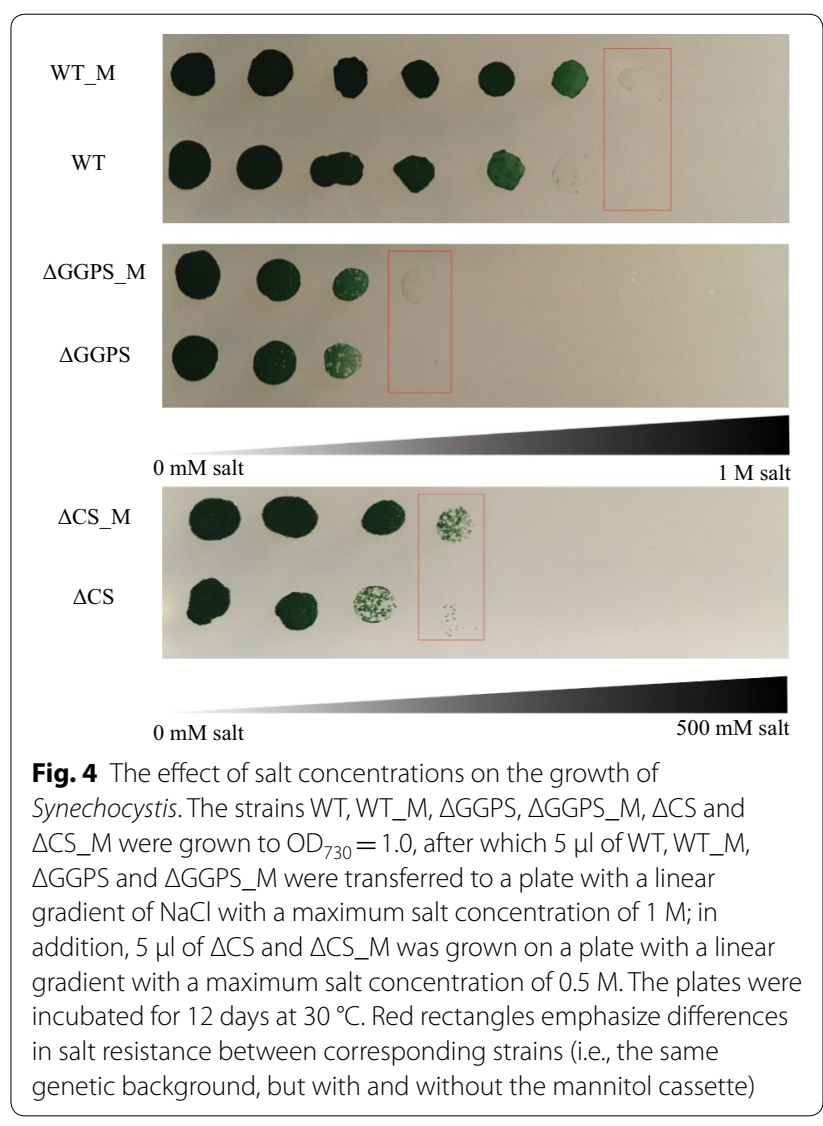

either glycosyl-glycerol (in the $\triangle$ GGPS strain), or both glycosyl-glycerol and sucrose (in the $\triangle \mathrm{CS}$ strain), was deleted, cells displayed a lower salt tolerance relative to the WT strain. This further supports the functionality of the native compatible solutes to resist salt stress. In 
the $\Delta$ CS_M mutant, mannitol is the only (known) compatible solute remaining. Indeed, mannitol production helped the $\triangle$ CS_M mutant to cope with higher salt stress (Fig. 4). Accordingly, we anticipated that mannitol production in Synechocystis can be stabilized via salt stress; yet this still needed to be directly tested.

\section{Salt stress stabilized mannitol production during prolonged cultivations}

To test the (genetic) stability of mannitol production, a suitable $\mathrm{NaCl}$ concentration has to be selected. This salt concentration should partially inhibit cell growth, but should still allow cells to replicate at a certain rate, such that reverting cells that may arise, but should not be able to take over the population in a relatively short time. Hence, we decided to first determine the growth rate of all the background strains under different $\mathrm{NaCl}$ concentrations in a 96-well plate growth assay (Fig. 5a and Additional file 1: Figure S1). As depicted in Fig. 5a, the results obtained showed that the growth rate of the WT strain was hardly affected, at least up to $400 \mathrm{mM} \mathrm{NaCl}$, and then its growth rate gradually decreased until the growth was completely arrested at $500 \mathrm{mM} \mathrm{NaCl}$. For the $\triangle$ GGPS strain, which lacks the main native compatible solute glucosyl-glycerol, the growth rate slightly decreased at $350 \mathrm{mM} \mathrm{NaCl}$ and suddenly dropped sharply to zero at $400 \mathrm{mM} \mathrm{NaCl}$, and the same trend is observed for the $\triangle \mathrm{CS}$. For this latter strain, the growth rate already decreased at $200 \mathrm{mM} \mathrm{NaCl}$ and went to zero at $300 \mathrm{mM} \mathrm{NaCl}$.

Based on these results, to check the stability of mannitol production during prolonged cultivation, we decided to select the $\mathrm{NaCl}$ concentration that allows $70 \%$ of the maximum growth rate. We decided to leave out the $\triangle$ GGPS strain, because of possible contribution of sucrose to the salt tolerance of the cells. Hence, $420 \mathrm{mM}$ and $200 \mathrm{mM} \mathrm{NaCl}$ were the salt concentrations chosen to test the WT_M and $\triangle \mathrm{CS} \_M$ strains, respectively.

Mannitol production for the WT_M and $\Delta$ CS_M strains was monitored with and without salt stress, in prolonged turbidostat cultivations. As shown in Fig. 5b, for both the WT_M and the $\Delta$ CS_M strain without salt stress, mannitol production was quickly lost until no mannitol could be measured after only 6 days of cultivation (13.5 generations). This further supports the argument that mannitol production is indeed unstable via common metabolic engineering strategies as previously observed in Synechococcus [9]. For the WT_M strain under salt stress, mannitol production gradually dropped to below the detection limit in 11 days (17.1 generations). This phenomenon is to be expected because native compatible solutes are likely to be preferred to resist salt stress, over the exogenous mannitol. In the $\Delta$ CS_M strain, mannitol will be the only compatible solute available to the cells to tolerate salt stress. Therefore, mannitol production is expected to be stable in this strain. This is in accordance with what we observed, as throughout the entire experiment (i.e., 12 days, 21.6 generations, see Fig. 5b), only a slight drop in mannitol production was observed in the $\triangle \mathrm{CS} \_\mathrm{M}$ strain, especially during days 6 and 7. We speculate that this slight decrease might be due to the fact that cells within the population are selected when mannitol production is fine-tuned to the amounts required by the environment imposed. These results
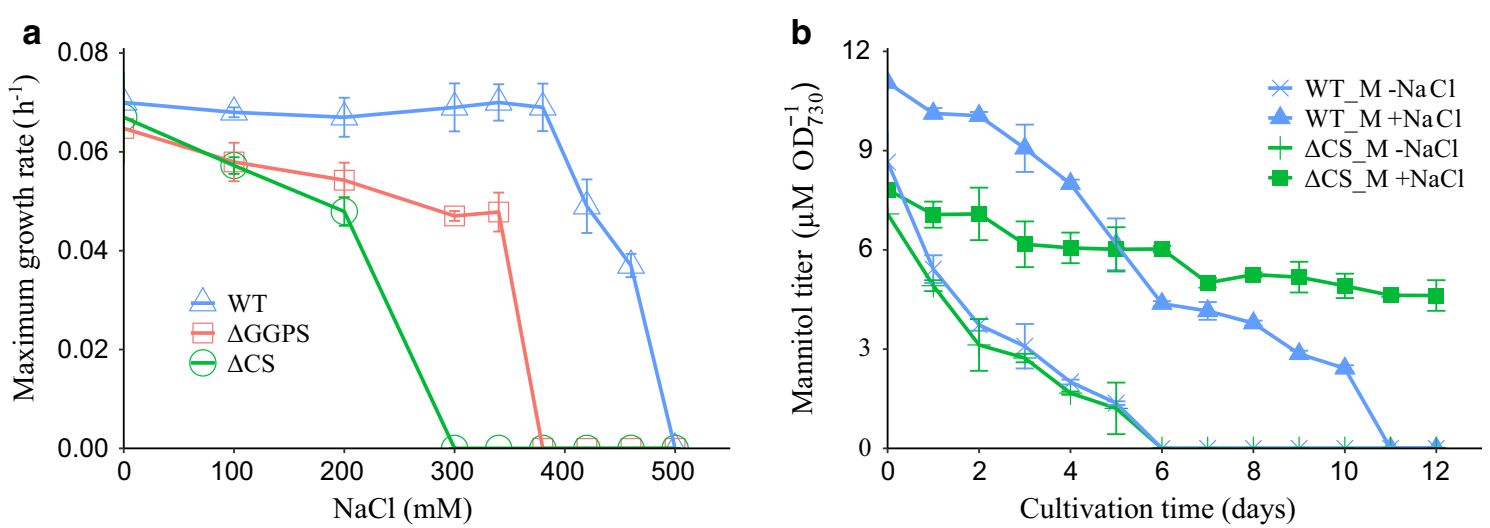

Fig. 5 Maximum growth rate for mutants under different salt concentration and mannitol production changed from mannitol producer under specific salt pressures in Multi-cultivator. a Growth rate of WT, $\triangle$ GGPS and $\triangle \mathrm{CS}$ as a function of salt concentration. Symbols: open triangles, WT; open squares, $\triangle \mathrm{GGPS}$; open circles, $\triangle \mathrm{CS}$. Values represent the average of biological duplicates (error bars represent standard deviation). b Mannitol production of WT_M and $\triangle C S \_M$ with and without salt stress in a Multi-cultivator. Symbols: crosses, WT_M grown in the presence of $420 \mathrm{mM}$ NaCl; triangles, WT_M grown without added salt; bars, $\triangle$ CS_M grown without added salt; squares, $\triangle C S$ CM grown in the presence of $200 \mathrm{mM}$ NaCl. Values represent the average of at least three biological replicates (error bars represent standard deviation) 
indicate that by exploiting salt stress as a selection pressure, one can stabilize mannitol production from $\mathrm{CO}_{2}$ in Synechocystis.

\section{Characterization of mutations that are selected by the phenotypic instability}

Mannitol production from WT_M and $\Delta C S \_M$ under no salt condition in the multi-cultivator was completely absent after only 13.5 generations, which corresponds to only 6 days of cultivation. Under $420 \mathrm{mM}$ salt, the WT_M produced decreasing amounts of mannitol until it completely ceased to do so after 17.1 generations in 11 days of cultivation. In sharp contrast, the $\triangle C S \_M$ under $200 \mathrm{mM}$ salt stress displayed remarkably stable mannitol production, which could still be observed after 12 days cultivation in the multi-cultivator, corresponding to over 21.6 generations. To clarify the molecular mechanism(s) behind the phenotypic instability of mannitol production, we decided to sequence the mannitol cassette of cultures derived from prolonged turbidostat cultivation experiments. From each strain and growth condition, 5 single colonies (i.e., 20 single colonies in total) were isolated when the mannitol productions from their responding culture were completely disrupted and their mannitol-producing cassette was amplified by PCR and sent for sequencing (Additional file 1: Table S2). Indeed, the results obtained showed that for those cultivations in which mannitol production was lost, various mutations could be detected, either leading to a truncated, non-functional, MtlD protein, or to impairment of its enzyme activity. All mutations found could be grouped in one of three types: single nucleotide insertions (SNI), single nucleotide deletions (SND) and single point mutations (PM) (see Additional file 1: Table S2 and Fig. 6). Among 14 mutations found in the strains that lost the ability to synthesize mannitol, 13 mutations occurred in $m t l D$ reading frame and one was in its (Ptrc1) promoter region. All these mutants of different mannitol producers cultured under various salt conditions were re-cultivated in the shake flasks, and none of them showed the ability of producing mannitol (data not shown). These results indicated that mannitol production was disrupted due to malfunction of MtlD and that mannitol-1-phosphate accumulation might be harmful to cells, even though the underlying mechanism is still unclear [11]. We know the MtlD protein contains two pfam domains, the mannitol dehydrogenase C-terminal domain (pfam08125) and mannitol dehydrogenase Rossmann domain (pfam01232). The Rossmann domain is specific for binding $\mathrm{NAD}(\mathrm{P})^{+}$and contains the conserved consensus motif $\mathrm{G}-\mathrm{x}-\mathrm{G}-\mathrm{x}-\mathrm{x}-\mathrm{G}$. The C-terminal domain contains conserved residues which bind the substrate fructose-6-phosphate, thus facilitating the catalytic reaction of the enzyme [24]. Our data revealed that three of the mutations were distributed over the Rossmann domain, 9 were in the C-terminal domain, including two identical mutations isolated independently, and one was in the linker region. It is important to note that one of the isolates from the $\Delta C S \_M$ population, cultured under no salt condition, showed no mutation while its culture had stopped producing mannitol within 6 days. This result suggests that though a very small proportion of this population might still have the ability to synthesize mannitol, its ratio of nonmutated over mutated cells must be too low to make detectable amounts of mannitol by enzymatic assay.

In sharp contrast, no mutation in the mannitol cassette was found in the $\Delta C S \_M$ strain during the entire prolonged cultivation under salt stress. This observation corroborates the result that cells also maintained a continuous mannitol producing phenotype under such conditions (Fig. 6a) and leads us to conclude that mannitol production in Synechocystis can be stabilized by salt stress, in mutants lacking the pathways for synthesis of the two endogenous osmoprotectants. In addition, by purposely weakening the native abilities of cells to tolerate salt stress $[25,26]$, this strategy could allow more mannitol to be synthesized to compensate the loss of salt tolerance ability. Such could allow that mannitol production could be even further sustainably enhanced.

\section{Conclusions}

Mannitol production was achieved in a freshwater cyanobacterium, a derivative of Synechocystis, this time conferring the producing strain with increased salt tolerance. With this approach we have tackled the major hurdle to mannitol production in cyanobacteria-genetic instability-by specifically aligning the production of a target compound with a fitness gain for the producing cells. Given the osmoprotectant properties of mannitol, we targeted the native osmotic stress response of Synechocystis, by replacing the synthesis of the endogenous compatible solutes by mannitol production. This strategy has been shown to be very successful, resulting in a production system for mannitol directly from $\mathrm{CO}_{2}$ with increased stability. The principle developed here, i.e., of coupling product formation to increased fitness under mild stress conditions, can potentially be applied to other host/product combinations as well. In future work it is important to take this principle of product-mediated stress protection already into account during the metabolic engineering design stage, as this engineering is likely to involve the identification and deletion of native stress response genes. 

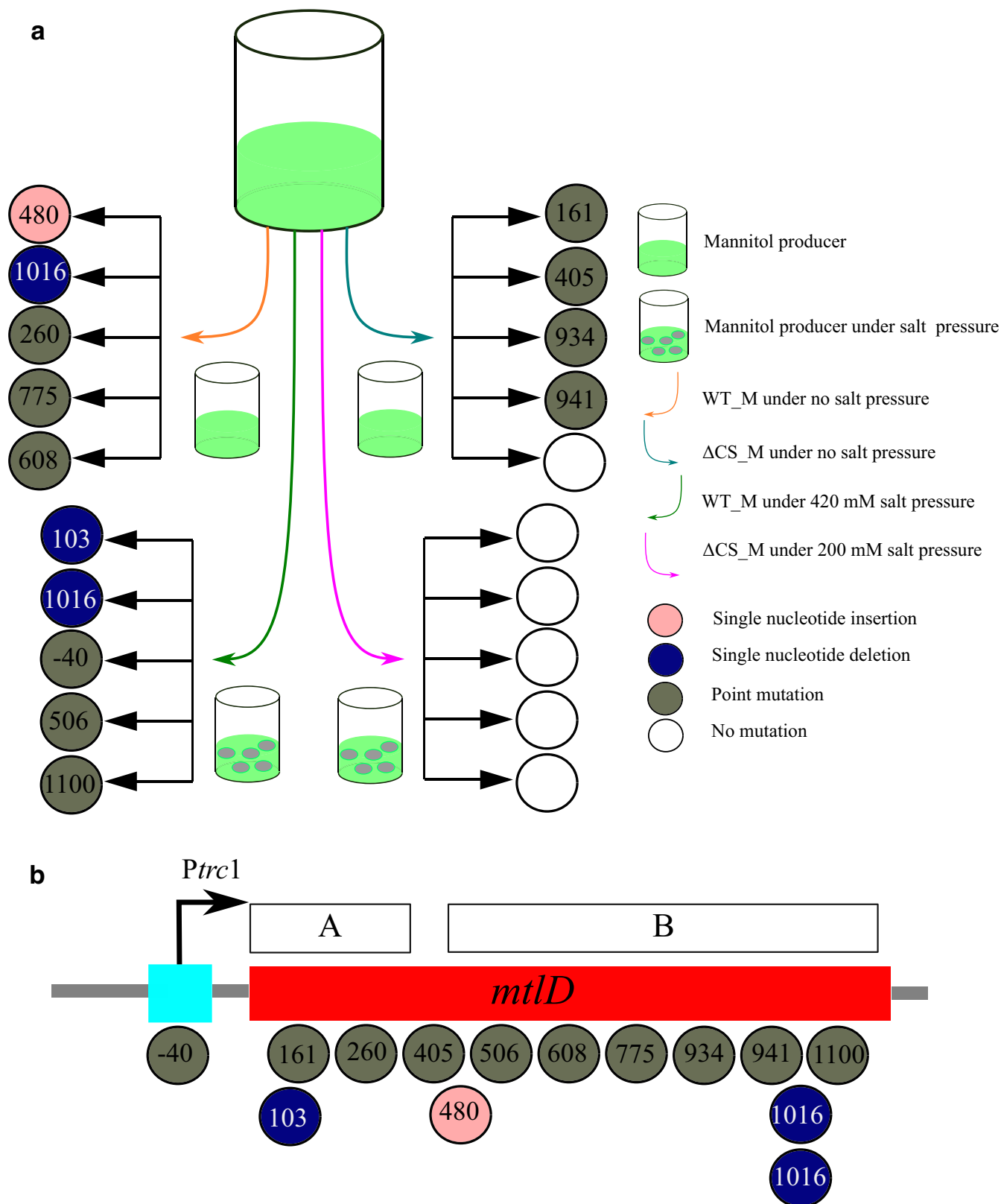

Fig. 6 Characterization of mutations underpinning phenotypic instability surrounding mannitol production under non-stabilizing conditions in Multi-cultivator. a Schematic representation on the analysis of genetic stability of mannitol producers in the Multi-cultivator. The colored arrows in the center refer to the four combinations of a relevant strain (i.e., WT and $\triangle C S \_M$ ) plus cultivation condition (i.e., in the presence and absence of $\mathrm{NaCl}$ ). Mannitol production capacity of WT_M and $\triangle \mathrm{CS} \_M$ under no salt condition was completely eliminated within only 6 days. WT_M produces decreasing amounts of mannitol, until it ceases to do so, during the first 10 days while under $420 \mathrm{mM}$ salt. The mannitol production from $\triangle C S \_M$ under $200 \mathrm{mM}$ salt was found to be the most stable, which could still be detected after 12 days. The grey ovals in green color indicate salt pressure. For further details, see Results and Discussion. $\mathbf{b}$ Schematic overview of all mutations observed in the mt/D-part of the mannitol cassette of the mannitol-producing strains. The numbers in the circles refer to the position of a mutation in the promoter region or in the reading frame of MtID. "A" in the bar above the reading frame of MtID represents the Rossmann domain, and "B" the c-terminal domain. In MtID, the two domains are linked via a linker region 


\section{Methods}

\section{Strains and culture conditions}

Strains of E. coli were grown on Lysogeny Broth (LB) liquid medium at $37{ }^{\circ} \mathrm{C}$ in a shaking incubator at $200 \mathrm{rpm}$, or on the solid LB plates with $1.5 \%$ agar. Antibiotics were added to LB liquid medium or to solid plates, with the appropriate concentration as follows: kanamycin $\left(50 \mu \mathrm{g} \mathrm{mL}^{-1}\right)$ or ampicillin $\left(100 \mu \mathrm{g} \mathrm{mL}{ }^{-1}\right)$, either separately or in combination.

Synechocystis, a glucose-tolerant wild type, obtained from D. Bhaya, University of Stanford, Stanford CA, was cultivated in a modified BG-11 medium enriched with 25 mM PIPPS buffer ( $\mathrm{pH}$ 8.0) [27] at $30^{\circ} \mathrm{C}$, either in a shaking incubator at $120 \mathrm{rpm}$, or on solid BG-11 plates, supplemented with $1.5 \%$ agar and $0.3 \%(\mathrm{w} / \mathrm{v})$ sodium thiosulphate. The cells were grown with white light of moderate intensity $\left(\sim 50 \mu \mathrm{mol}\right.$ photons $\left.\mathrm{m}^{-2} \mathrm{~s}^{-1}\right)$, except when indicated. To construct Synechocystis mutants, kanamycin or nickel was added separately into BG-11 liquid medium or solid plates, with the appropriate concentration as follows: kanamycin $\left(50 \mu \mathrm{g} \mathrm{mL}^{-1}\right)$ and/or nickel $(20 \mu \mathrm{M})$. The culture density was monitored by determining the optical density at a wavelength of $730 \mathrm{~nm}\left(\mathrm{OD}_{730}\right)$.

\section{Gene synthesis with codon optimization}

The gene sequence of mannitol-1-phosphate-5-dehydrogenase $(m t l D)$ from $E$. coli (NCBI Reference Sequence: NC_000913.3) and mannitol-1-phosphatase $(m 1 p)$ from Eimeria tenella (NCBI Reference Sequence: AF032462.1) was taken directly from the NCBI database. Codon optimization was performed based on the codon usage table compiled for Synechocystis (https://www.kazusa.or.jp/ codon/). The genes were synthesized by GenScript Biotech Corp, and were ligated to the pHKH and pUC57 plasmids, respectively [28] resulting in pHKHmtlD and pUCm1p.

\section{Plasmids and Synechocystis mutant construction}

To obtain marker-less deletion strains, the genes encoding sucrose phosphate synthase (sps) and glucosylglycerol phosphate synthase $(g g p S)$ in the genome of Synechocystis were deleted with a counter-selection approach [29]. For each gene deletion, two plasmids are needed: the first one contains only the upstream and the downstream sequences of the region to be deleted, hereafter referred as the homologous regions, while the second plasmid contains an extra selection cassette flanked by both homologous regions. The selection cassette consists of a gene conferring kanamycin resistance to the host, as well as a toxic gene $(m a z F)$, under transcriptional control of the tightly regulated nickel-inducible promoter PnrsB. To construct each of the two plasmids, $\sim 1 \mathrm{~kb}$ of each upstream and downstream homologous region of either sps or $g g p S$ was individually PCR-amplified from the genome of Synechocystis, using Herculase II Fusion DNA Polymerase (Agilent Technologies). After gel purification, each set of fragments of an upstream and downstream homologous region was fused by overlap PCR, and the entire fused fragment was then further amplified by PCR. After the fused fragment was gel purified, an extra adenosine was added to the $3^{\prime}$ ends of these fragments and it was then ligated to the pFL-AN-T vector [30], resulting in plasmids pFL-AN2 $(\Delta s p s)$ and pFL-AN4 $(\Delta g g p S)$, respectively. Because an $\mathrm{Xbal}$ restriction site was introduced via the primers during overlap PCR, the selection cassette, if provided with an $\mathrm{XbaI}$ site on both ends, can be easily inserted into pFL-AN2 $(\Delta s p s)$ and pFL-AN4 $(\Delta g g p S)$, resulting in pFL-AN1 $(\Delta s p s)$ and pFLAN3 $(\Delta g g p S)$, respectively.

The $m t l D$ and $m 1 p$ genes were PCR amplified from pHKHmtlD and pUCm1p, respectively. Initially, we attempted to clone these genes using $E$. coli as a shuttle host. To correctly express mtlD in Synechocystis, the weaker promoter PnrsB was used to control the expression level of $m t l D$ in Synechocystis, but repeatedly failed. This result surprised us, as PnrsB is from the nickel response system (nrs [31]), and is regarded as one of the weakest promoters in this host in the absence of an inducer. This result indicated the difficulty of high expression levels of MtlD, and that this might become a bottleneck for the synthesis of large amounts of mannitol in Synechocystis. So we decided to bypass the usage of a shuttle host. Instead we chose to fuse these two fragments together with the kanamycin resistance gene, and the upstream and downstream homologous regions of slr0168, via overlap PCR. The resulting mannitol cassette plus resistance marker was placed under control of the Ptrc1 promoter. The fused fragment was sequenced to check for the absence of mutations and then used directly for transformation of the chromosome of Synechocystis at the neutral site present in locus slr0168.

It takes two rounds of natural transformation of Synechocystis to achieve a markerless gene deletion of either sps or ggpS. The first round is to fully integrate the selection cassette into the chromosome through homologous recombination, while the second round is to completely remove the selection cassette. The method used for natural transformation was essentially as described previously [32]. In brief, $1 \mathrm{ml}$ Synechocystis cultures grown in a shake flask to an $\mathrm{OD}_{730}$ of $\sim 0.4$ were harvested and concentrated by centrifugation at $5000 \mathrm{rpm}$ for $5 \mathrm{~min}$ to a volume of $200 \mu \mathrm{L}$. Then, plasmid was added to the concentrated cells at $10 \mu \mathrm{g} \mathrm{mL} \mathrm{m}^{-1}$, followed by $5 \mathrm{~h}$ ' incubation in moderate white light, in a shaking incubator at $150 \mathrm{rpm}$. After incubation, cells were spread onto a 
commercial membrane (Pall Corporation, USA) resting on a BG-11 plate without antibiotic pressure. After a 24-h incubation in the $30{ }^{\circ} \mathrm{C}$ incubator under constant white light illumination, the membrane was transferred onto a new BG-11 plate with $50 \mu \mathrm{g} \mathrm{mL}^{-1}$ kanamycin. Single colonies appeared after approximately 12 days. The segregation status of mutants was confirmed by PCR, using the appropriate primers. When a mutant was confirmed to be fully segregated, a second round of transformation with a plasmid containing only the upstream and downstream homologous region was performed. The selection was then based on the resistance to nickel as only the colonies with the selection cassette fully removed can survive on the plates with nickel. The protocol for transformation of the DNA fragment containing the mannitol cassette and the kanamycin resistance fragment integrated at the slr0168 site was similar to the protocol mentioned above. Full segregation of this construct was achieved by propagations in the presence of kanamycin. All the mutants were confirmed by PCR and the primers that were used are listed in Additional file 1: Table S3. The confirmed mutants were routinely stored at $-80{ }^{\circ} \mathrm{C}$ in BG-11 medium supplemented with $20 \%$ (v/v) glycerol.

\section{Growth rate determination under salt stress}

To measure the growth rate for each strain under salt stress, we monitored the growth of each strain in a 96-well plate under a range of $\mathrm{NaCl}$ concentrations, from 0 to $500 \mathrm{mM}$. A preculture was prepared by inoculating cells from glycerol stocks directly into shake flasks containing liquid BG-11 medium and cultivated in the incubator with shaking of $120 \mathrm{rpm}$ under continuously white light of moderate intensity $\left(\sim 50 \mu \mathrm{mol}\right.$ photons $\left.\mathrm{m}^{-2} \mathrm{~s}^{-1}\right)$. Once the precultures reached $\mathrm{OD}_{730}=1$, a total volume of $1 \mathrm{~mL}$ of a pre-culture was harvested and inoculated in each well of a 48-well plate, supplemented with $50 \mathrm{mM}$ $\mathrm{NaHCO}_{3}$ plus increasing salt concentrations, ranging from 0 to $500 \mathrm{mM}$. After 2 days, the final optical density of the cultures in the 48-well plate was measured using a SPECTROstar Nano Microplate Photometer (BMG LABTECH GmbH, Germany) at $730 \mathrm{~nm}$. To initiate experiments with a 96-well plate, pre-cultures acclimated to the corresponding salt stress in the 48-well plate were used for inoculation. This is to prevent the prolonging of the lag-phase of growth, resulting from the addition of salt, such that the same stage of cell growth under each condition can be extracted from the data for growth rate calculation. Pre-cultures were then diluted using $50 \mathrm{mM}$ $\mathrm{NaHCO}_{3}$ and the respective concentration of $\mathrm{NaCl}$ in BG-11 to an initial OD of 0.05. Plates were incubated under constant white light illumination, with shaking at $600 \mathrm{rpm}$. Growth was monitored every $2 \mathrm{~h}$ within the 36 -h incubation in the plate reader, to reliably calculate the maximum growth rate under each condition, the first 6 consecutive data points (from time 0 to $10 \mathrm{~h}$ ) were used by fitting a linear function through the natural logarithm of the OD. The slope of the linear function was computed and designated as the growth rate. A representative set of growth curves in the 96 well plate together with the data points for growth rate calculation for all the strains is presented in Additional file 1: Fig. S1.

\section{Plate assay with a linear $\mathrm{NaCl}$ gradient}

The method for making linear salt gradient plates has been described previously by [33]. In brief, BG-11 media containing agar, with and without $\mathrm{NaCl}$ (either $0.5 \mathrm{M}$ or $1 \mathrm{M}$ ), were individually prepared. When making linear salt gradient plates, one side of a square petri dish was lifted and the plate was filled with BG-11 agar without $\mathrm{NaCl}$. After the agar solidified, the plate was placed in a horizontal position and BG-11 agar with $\mathrm{NaCl}$ was poured on top of the first layer. To test the salt tolerance of all the mutants, the cells in Synechocystis cultures were first counted using a Casy 1 Model TTC cell counter (Schärfe System GmbH, Reutlingen, Germany) with a $60-\mu \mathrm{m}$ diameter capillary, and diluted to a total cell number of 12,500 cells $\mu \mathrm{L}^{-1}$. A $5 \mu \mathrm{L}$ culture from each mutant, grown with $200 \mathrm{mM}$ salt, was spotted on the linear gradient plates containing a 0 to $0.5 \mathrm{M}$ or a 0 to $1 \mathrm{M} \mathrm{NaCl}$ concentration gradient. Visible, green colonies appeared within 1 week.

\section{Measurements of extracellular mannitol concentrations}

Extracellular mannitol concentrations were determined in the supernatant collected from Synechocystis cultures using a D-Mannitol-L-Arabitol Assay Kit (Megazyme) [9]. Cells from shake-flask cultures were removed by centrifugation at $12,000 \mathrm{rpm}$ for $1 \mathrm{~min}$. Then, $100 \mu \mathrm{L}$ of the supernatant samples was used for mannitol measurement according to manufacturer's instructions. In this assay, the conversion of the mannitol present in the sample to mannose-catalyzed by mannitol dehydrogenaseis stoichiometrically coupled to the conversion of $\mathrm{NAD}^{+}$ to NADH. This leads to an increase of absorbance at $340 \mathrm{~nm}$ that can be measured using a plate reader (BMG FLUOstar OPTIMA Microplate Reader). For mannitol quantification, the assay was calibrated with a standard curve (from 3 to $100 \mu \mathrm{M}$ mannitol) obtained under the same conditions.

\section{Turbidostat cultivation}

The phenotypic stability of mannitol-producing strains was studied with the turbidostat mode of a Multi-Cultivator (MC1000-OD, PSI, Czech Republic). In this cultivation mode, Synechocystis populations can be kept at a fixed biomass density by continuously diluting cultures 
with fresh BG-11 medium without antibiotic, while simultaneously taking out an identical volume of cultured cells. Accordingly, cells in a turbidostat are under continuous selection for maximal specific growth rate. For this we used a modified Multi-Cultivator with additional pumps (Reglo ICC, ISMATEC, Germany) and controlled by the "pycultivator" software package [13]. Pre-cultured cells were transferred into 8 independent cylindrical vessels of a multi-cultivator, filled with BG-11 medium with $0,200 \mathrm{mM}$ or $420 \mathrm{mM} \mathrm{NaCl}$ to an initial $\mathrm{OD}_{730}$ of 0.05 . $\mathrm{OD}_{730}$ was measured every $5 \mathrm{~min}$. Once the threshold of $\mathrm{OD}_{730}$ was reached $\left(\mathrm{OD}_{730}=1\right)$ cell cultures were automatically diluted by $5 \%(\mathrm{v} / \mathrm{v})$ with fresh BG-11 medium and the same volume of culture as the volume of medium just added, was discarded; all under control of "pycultivator". All the cultures in the Multi-Cultivator were exposed to continuous white light with an intensity of $100 \mu \mathrm{mol}$ photons $\mathrm{m}^{-2} \mathrm{~s}^{-1} \mathrm{OD}^{-1}$. The genetic stability of each strain was assessed by mannitol production. Growth rate was calculated by fitting a linear function through the natural logarithm of the $\mathrm{OD}_{730}$ during each "growth-dilution" cycle. Samples for extracellular mannitol quantification were periodically taken during the cultivation period. The variation of growth rate and mannitol productivity throughout the whole experiment was then calculated relative to the initial values obtained at the beginning of each specific experiment.

\section{Sequencing of the mannitol cassette}

To check the sequence, the mannitol cassette for the occurrence of (a) spontaneous mutation(s), single colonies from each independent Multi-Cultivator culture were first isolated. This is achieved by taking $5 \mu \mathrm{L}$ of culture and re-streaking the cells on BG-11 agar plates. After picking a single colony and inoculating it into liquid BG-11 medium, genomic DNA was extracted as previously described [34] and used as a template to amplify the mannitol cassette by PCR, using the high-fidelity Herculase II Fusion DNA Polymerase. The PCR product was then purified using MSB Spin PCRapace (STRATEC Molecular, Germany) and sent for sequencing (Macrogen) using the primers listed in Additional file 1: Table S3.

\section{Supplementary information}

Supplementary information accompanies this paper at https://doi. org/10.1186/s13068-020-01755-3.

Additional file 1. Additional tables and figure.

\section{Acknowledgements}

We thank Tania Chroumpi for technical support in the initial experiments.

\section{Authors' contributions}

WW has performed most of the strain engineering and cultivation of strains under different regimes supervised by WD and FBS. RPG constructed some of the deletion strains and conducted preliminary characterization assays of their salt tolerance under the supervision of AW. WW and WD analyzed the data obtained supervised by FBS. AW, KJH and FBS conceived the project. WW and WD wrote the first draft of the manuscript assisted by FBS and KJH. All authors read and approved the final manuscript.

\section{Funding}

The work of W.W. was supported by the China Scholarship Council. F.B.S. and W.D. were supported by the European Union's EFRO grant'Kansen voor West II' The Netherlands Organization for Scientific Research (NWO) supported F.B.S. through Solar-2-product Grant 733000005 and from the European Union's Horizon 2020 research and innovation programme under Grant agreement No. 760994 (ENGICOIN project). The funders had no role in study design, data collection and analysis, decision to publish, nor preparation of the manuscript.

\section{Availability of data and materials}

The datasets generated during this study are included in this published article and its additional file.

\section{Ethics approval and consent to participate}

Not applicable.

\section{Consent for publication}

Not applicable.

\section{Competing interests}

Klaas J. Hellingwerf and Aniek van der Woude have active roles as the scientific advisor and lead scientist, respectively, within Photanol B.V., a University of Amsterdam spin-off company aiming at commercializing sustainable applications with cyanobacteria. The authors declare that the research was conducted in the absence of any commercial or financial relationship that could be construed as a potential conflict of interest.

\section{Author details}

${ }^{1}$ Molecular Microbial Physiology Group, Swammerdam Institute for Life Sciences, Faculty of Science, University of Amsterdam, Science Park 904, 1098 XH Amsterdam, The Netherlands. ${ }^{2}$ Photanol B.V, Matrix V, Science Park 406, 1098 XH Amsterdam, The Netherlands. ${ }^{3}$ Present Address: NIOZ Royal Netherlands Institute for Sea Research, Department of Marine Microbiology and Biogeochemistry, Utrecht University, P.O. Box 59, Den Burg, Texel, 1790 AB Utrecht, The Netherlands.

Received: 13 March 2020 Accepted: 23 June 2020

Published online: 02 July 2020

\section{References}

1. Saha BC, Racine FM. Biotechnological production of mannitol and its applications. Appl Microbiol Biotechnol. 2011;89(4):879-91.

2. Angermayr SA, Gorchs Rovira A, Hellingwerf KJ. Metabolic engineering of cyanobacteria for the synthesis of commodity products. Trends Biotechnol. 2015;33(6):352-61.

3. Dexter J, Fu P. Metabolic engineering of cyanobacteria for ethanol production. Energy Environ Sci. 2009;2(8):857-64.

4. Angermayr SA, van der Woude AD, Correddu D, Vreugdenhil A, Verrone V, Hellingwerf KJ. Exploring metabolic engineering design principles for the photosynthetic production of lactic acid by Synechocystis sp. PCC6803. Biotechnol Biofuels. 2014;7(1):99.

5. Englund E, Shabestary K, Hudson EP, Lindberg P. Systematic overexpression study to find target enzymes enhancing production of terpenes in Synechocystis PCC 6803, using isoprene as a model compound. Metab Eng. 2018;49:164-77.

6. Savakis $\mathrm{P}$, Hellingwerf KJ. Engineering cyanobacteria for direct biofuel production from $\mathrm{CO}_{2}$. Curr Opin Biotechnol. 2015;1(33):8-14. 
7. Choi Y-N, Park JM. Enhancing biomass and ethanol production by increasing NADPH production in Synechocystis sp. PCC 6803. Bioresour Technol. 2016;213:54-7.

8. Joseph A, Aikawa S, Sasaki K, Tsuge Y, Matsuda F, Tanaka T, et al. Utilization of lactic acid bacterial genes in Synechocystis sp. PCC 6803 in the production of lactic acid. Biosci Biotechnol Biochem. 2013;77(5):966-70.

9. Jacobsen $\mathrm{JH}$, Frigaard N-U. Engineering of photosynthetic mannitol biosynthesis from $\mathrm{CO}_{2}$ in a cyanobacterium. Metab Eng. 2014;1 (21):60-70.

10. Madsen MA, Semerdzhiev S, Amtmann A, Tonon T. Engineering Mannitol Biosynthesis in Escherichia coli and Synechococcus sp. PCC 7002 Using a Green Algal Fusion Protein. ACS Synth Biol. 2018;7(12):2833-40.

11. Sand M, Rodrigues M, González JM, de Crécy-Lagard V, Santos H, Müller $\checkmark$, et al. Mannitol-1-phosphate dehydrogenases/phosphatases: a family of novel bifunctional enzymes for bacterial adaptation to osmotic stress. Environ Microbiol. 2015;17(3):711-9.

12. Du W, Burbano PC, Hellingwerf KJ, dos Santos FB. Challenges in the application of synthetic biology toward synthesis of commodity products by cyanobacteria via "direct conversion."In: Synthetic Biology of Cyanobacteria. Springer; 2018. p. 3-26.

13. Du W, Jongbloets JA, van Boxtel C, Pineda Hernández H, Lips D, Oliver BG, et al. Alignment of microbial fitness with engineered product formation: obligatory coupling between acetate production and photoautotrophic growth. Biotechnol Biofuels. 2018;11(1):38.

14. Chaturvedi $V$, Bartiss ANN, Wong B. Expression of bacterial $m t / D$ in Saccharomyces cerevisiae results in mannitol synthesis and protects a glycerol-defective mutant from high-salt and oxidative stress. J Bacteriol. 1997;179(1):157-62

15. Kirsch F, Klähn S, Hagemann M. Salt-regulated accumulation of the compatible solutes sucrose and glucosylglycerol in cyanobacteria and its biotechnological potential. Front Microbiol. 2019;10:2139.

16. Keshari N, Gugger M, Zhu T, Lu X. Compatible solutes profiling and carbohydrate feedstock from diversified cyanobacteria. Algal Res. 2019:43:101637.

17. Hagemann M. Genomics of salt acclimation: synthesis of compatible solutes among cyanobacteria. In: Advances in botanical research. Elsevier; 2013. p. 27-55.

18. Thiel K, Patrikainen P, Nagy C, Fitzpatrick D, Pope N, Aro E-M, et al. Redirecting photosynthetic electron flux in the cyanobacterium Synechocystis sp. PCC 6803 by the deletion of flavodiiron protein Flv3. Microb Cell Fact. 2019:18(1):189.

19. Klähn S, Hagemann M. Compatible solute biosynthesis in cyanobacteria Environ Microbiol. 2011;13(3):551-62.

20. Pade N, Hagemann M. Salt acclimation of cyanobacteria and their application in biotechnology. Life. 2015;5(1):25-49.

21. Ferenci T, Kornberg HL. The utilization of fructose by Escherichia coli Properties of a mutant defective in fructose 1-phosphate kinase activity. Biochem J. 1973;132(2):341-7.
22. Berkowitz D. D-Mannitol utilization in Salmonella typhimurium. J Bacteriol. 1971;105(1):232-40.

23. Kaneko T, Sato S, Kotani H, Tanaka A, Asamizu E, Nakamura Y, et al. Sequence Analysis of the Genome of the Unicellular Cyanobacterium Synechocystis sp. Strain PCC6803. II. Sequence Determination of the Entire Genome and Assignment of Potential Protein-coding Regions. DNA Res. 1996;3(3):109-36.

24. Wyatt TT, Van Leeuwen MR, Wösten HAB, Dijksterhuis J. Mannitol is essential for the development of stress-resistant ascospores in Neosartorya fischeri (Aspergillus fischeri). Fungal Genet Biol. 2014;64:11-24.

25. Nikkinen H-L, Hakkila K, Gunnelius L, Huokko T, Pollari M, Tyystjärvi T. The SigB $\sigma$ factor regulates multiple salt acclimation responses of the cyanobacterium Synechocystis sp. PCC 6803. Plant Physiol. 2012;158(1):514-23.

26. Hu L, He J, Dong M, Tang X, Jiang P, Lei A, et al. Divergent metabolic and transcriptomic responses of Synechocystis sp. PCC 6803 to salt stress after adaptive laboratory evolution. Algal Res. 2020;47:101856.

27. van Alphen P, Abedini NH, dos Santos F, Hellingwerf KJ. Increasing the photoautotrophic growth rate of Synechocystis sp. PCC 6803 by identifying the limitations of its cultivation. Biotechnol J. 2018;13(8):1700764.

28. van der Woude AD, Gallego RP, Vreugdenhil A, Veetil VP, Chroumpi T, Hellingwerf KJ. Genetic engineering of Synechocystis PCC6803 for the photoautotrophic production of the sweetener erythritol. Microb Cell Fact. 2016;15(1):60

29. Cheah YE, Albers SC, Peebles CAM. A novel counter-selection method for markerless genetic modification in Synechocystis sp. PCC 6803. Biotechnol Prog. 2013;29(1):23-30.

30. Zhu T, Xie X, Li Z, Tan X, Lu X. Enhancing photosynthetic production of ethylene in genetically engineered Synechocystis sp. PCC 6803. Green Chem. 2015;17(1):421-34

31. López-Maury L, García-Domínguez M, Florencio FJ, Reyes JC. A twocomponent signal transduction system involved in nickel sensing in the cyanobacterium Synechocystis sp. PCC 6803. Mol Microbiol. 2002;43(1):247-56

32. Kufryk Gl, Sachet M, Schmetterer G, Vermaas WFJ. Transformation of the cyanobacterium Synechocystis sp. PCC 6803 as a tool for genetic mapping: optimization of efficiency. FEMS Microbiol Lett. 2002;206(2):215-9.

33. Creasy A, Barker G, Carta G. Systematic interpolation method predicts protein chromatographic elution with salt gradients, $\mathrm{pH}$ gradients and combined salt/pH gradients. Biotechnol J. 2017;12(3):1600636.

34. Savakis P, Tan X, Qiao C, Song K, Lu X, Hellingwerf KJ, et al. S/r1670 from Synechocystis sp. PCC 6803 is required for the re-assimilation of the osmolyte glucosylglycerol. Front Microbiol. 2016;7:1350.

\section{Publisher's Note}

Springer Nature remains neutral with regard to jurisdictional claims in published maps and institutional affiliations.
Ready to submit your research? Choose BMC and benefit from:

- fast, convenient online submission

- thorough peer review by experienced researchers in your field

- rapid publication on acceptance

- support for research data, including large and complex data types

- gold Open Access which fosters wider collaboration and increased citations

- maximum visibility for your research: over 100M website views per year

At BMC, research is always in progress.

Learn more biomedcentral.com/submissions 